\title{
Online Fault Detection Based on Typicality and Eccentricity Data Analytics
}

\author{
Bruno Sielly Jales Costa*, Clauber Gomes Bezerra ${ }^{\dagger}$, Luiz Affonso Guedes ${ }^{\ddagger}$ and Plamen Parvanov Angelov ${ }^{\S}$ \\ *Campus Natal - Zona Norte \\ Federal Institute of Rio Grande do Norte - IFRN \\ Natal, Brazil \\ Email: bruno.costa@ifrn.edu.br \\ †Campus EaD \\ Federal Institute of Rio Grande do Norte - IFRN \\ Natal, Brazil \\ Email: clauber.bezerra@ifrn.edu.br \\ $\ddagger$ Department of Computer Engineering and Automation \\ Federal University of Rio Grande do Norte - UFRN \\ Email: affonso@dca.ufrn.br

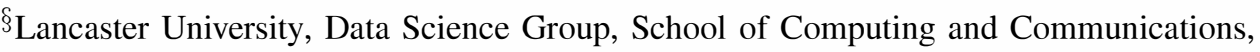 \\ Lancaster LA1 4WA, United Kingdom \\ Chair of Excellence, Carlos III University, Madrid, Spain \\ Email: p.angelov@lancaster.ac.uk
}

\begin{abstract}
Fault detection is a task of major importance in industry nowadays, since that it can considerably reduce the risk of accidents involving human lives, in addition to production and, consequently, financial losses. Therefore, fault detection systems have been largely studied in the past few years, resulting in many different methods and approaches to solve such problem. This paper presents a detailed study on fault detection on industrial processes based on the recently introduced eccentricity and typicality data analytics (TEDA) approach. TEDA is a recursive and non-parametric method, firstly proposed to the general problem of anomaly detection on data streams. It is based on the measures of data density and proximity from each read data point to the analyzed data set. TEDA is an online autonomous learning algorithm that does not require a priori knowledge about the process, is completely free of user- and problem-defined parameters, requires very low computational effort and, thus, is very suitable for real-time applications. The results further presented were generated by the application of TEDA to a pilot plant for industrial process.
\end{abstract}

\section{INTRODUCTION}

With the growing demand for more productive processes, complexity and size of industrial plants are increasing considerably, making its maintenance more and more difficult. Industrial equipment are subject to natural degradation signs over time, such as exhaustion, dirt, corrosion, cleavage and damage caused by operators. The appearance of any of these signs make the plant more susceptible to faults during operation.

A fault can be defined as an unallowed deviation of at least one of the features or parameters of a system from an acceptable/usual/standard condition [1]. In general, a fault is an unexpected alteration on the behavior of a system that can lead it to a critical situation.

In controlled dynamic systems, if no preventive action is taken, the appearance of faults become inevitable, which may lead the process to deviate from its normal state of operation, since that the system is not able to efficiently handle the fault. If the fault is not promptly corrected by a proper action, the system can reach an emergency situation, often resulting in a series of problems, such as unscheduled stoppages, production losses, reduction of equipment lifespan and accidents with consequences to human life and the environment. For example, the accident occurred in P-36 platform of PETROBRAS at the year of 2001 resulted in the loss of 11 human lives, in addition to environmental pollution and financial losses of about five billion dollars [2].

Very often, the process of fault detection in industrial environments is still manual and dependent on the expertise of the operator, which makes the process imprecise and considerably slow. Moreover, due to the great complexity of the monitored systems, the work of operators is even more difficult. According to Venkatasubramanian et. al. [3], statistics show that about $70 \%$ of the industrial accidentes are caused by human errors.

However, the increasing demand for productivity and safety resulted in great academic effort in the past few years on fault detection area of study. Very often, different techniques are combined in fault detection and diagnosis algorithms. Among these techniques, one can mention statistical [4], [5], observer-based [6], [7], analytical redundancy-based [8], [9], fuzzy model-based [10], [11], neural network-based [12], [13], immune system-based methods [14], [15] and so on.

However, many of these techniques need a priori knowledge about the behavior of the process, requiring the use of mathematical models and/or user-defined parameters, limiting the applicability of such approaches to real industrial processes. Moreover, in a fault detection application, the data is frequently acquired from several different process measured 
variables, at each time instant $k$, and need to be processed in real-time. Therefore, one should expect that a fault detection system be able to handle large amounts of data, providing quick and reliable response with low computational effort [3].

Very recently, a new anomaly/outlier detection that meet most of the mentioned requirements was introduced by [16]. Typicality and Eccentricity Data Analytics (TEDA) works with the concepts of typicality and eccentricity of data samples, based on their distance to the whole data set. Moreover, TEDA is recursively calculated, which makes it very suitable for realtime applications. In this work, TEDA is used for the first time in a fault detection application using real industrial data.

The remainder of this paper is organized as follows: Section II reviews the general concepts of TEDA. In Section III, DAMADICS fault detection benchmark is presented. In Section IV, the obtained results are presented and discussed. Finally, Section V presents the conclusions and future work.

\section{TEDA}

Our current approach is based on recently introduced anomaly detection technique called Typicality and Eccentricity Data Analitics (TEDA). The TEDA framework builds upon the well-known Recursive Density Estimation (RDE) algorithm family [17], however, with major differences in its formulation. It was first published in [16] and, since then, has been applied to different classification problems [18], [19].

TEDA algorithm aims to generalize and avoid any restrictive assumptions inherited from traditional statistical methods and probability theory, such as independence of individual data samples from each other, inability to work with very large data sets and prior assumptions of data distribution (e.g. Gaussian) [16]. Traditional statistical approaches are often very suitable for random processes, however might violate or ignore the dependence of data in real processes, such as climate, physical, social, economic and so on.

Fault detection, i.e. the task of distinguish a faulty state from a normal state of operation, is a common and recurrent anomaly detection application in industry. The data from a real industrial process presents, in the vast majority of times, many characteristics that makes the use of traditional statistical approaches not to be suitable for industrial applications, although have been often used for many years [20], [21].

TEDA, thus, emerges as an alternative statistical framework that can work efficiently with any data other than pure random processes in which each observation is completely independent from the others [16]. TEDA is based on several new quantities that are based on the proximity analysis in the $n$-dimensional feature space. The term typicality used in TEDA is somewhat similar to the recently introduced term with the same name in [22] to describe "the extent to which objects are 'good examples' of a concept".

Let us have a data space $X \in \Re^{n}$, which consists of $\mathrm{n}$ dimensional data samples/observations/measurements. For this space, we can define a distance $d(x, y)$, where Euclidean, Mahalanobis or any other measures can be used. Then, let us consider the data samples as an ordered sequence $\left\{x_{1}, x_{2}, \ldots, x_{k}, \ldots\right\}, x_{i} \in R^{n}, i \in N$, where the index $k$ represents the time instant of the observation.
For the whole data set/stream, we can define sum distance $p i$ to some particular point $x \in X$, for each element up to the $k^{\text {th }}$ one:

$$
\pi^{k}(x)=\sum_{i=1}^{k} d\left(x, x_{i}\right)
$$

The eccentricity $\xi$ of the data sample $x$ at the time instant $k$ can be defined as [23]

$$
\begin{gathered}
\xi^{k}(x)=\frac{2 \pi^{k}(x)}{\sum_{i=1}^{k} \pi^{k}\left(x_{i}\right)}=2 \frac{\sum_{i=1}^{k} d\left(x, x_{i}\right)}{\sum_{i=1}^{k} \sum_{j=1}^{k} d\left(x_{i}, x_{j}\right)}, \\
k \geq 2, \quad \sum_{i=1}^{k} \pi^{k}(x)>0
\end{gathered}
$$

As a complement to the eccentricity, the typicality $\tau$ of the data sample $x$ at the time instant $k$ is also defined as [24]:

$$
\tau\left(x_{k}\right)=1-\xi^{k}(x)
$$

The eccentricity and typicality are both bounded [23]:

$$
\begin{gathered}
0 \leq \xi^{k}(x) \leq 1, \sum_{i=1}^{k} \xi^{k}\left(x_{i}\right)=2 \\
0 \leq \tau^{k}(x) \leq 1, \sum_{i=1}^{k} \tau^{k}\left(x_{i}\right)=k-2, \\
k \leq 2 \\
\sum_{i=1}^{k} \pi^{k}\left(x_{i}\right)>0
\end{gathered}
$$

Both values of typicality and eccentricity can be, as RDE, calculated recursively. It can be shown, that equation 2 can be derived as an exact as [19]

$$
\xi^{k}(x)=\frac{1}{k}+\frac{\left(\mu_{x}^{k}-x\right)^{T}\left(\mu_{x}^{k}-x\right)}{k\left[\sigma_{x}^{k}\right]^{2}}
$$

The values of mean $\mu_{x}^{k}$ and variance $\sigma_{x}^{k}$ can be calculated recursively by [19]

$$
\begin{gathered}
\mu_{x}^{k}=\frac{(k-1) \mu_{x}^{k-1}}{k}+\frac{x_{k}}{k}, \quad k \geq 1, \quad \mu_{x}^{0}=0 \\
\mu_{x^{T} x}^{k}=\frac{(k-1) \mu_{x^{T} x}^{k-1}}{k}+\frac{x_{k}^{T} x_{k}}{k}, \quad k \geq 1, \quad \mu_{x^{T} x}^{0}=0 \\
{\left[\sigma_{x}^{k}\right]^{2}=\mu_{x^{T} x}^{k}-\left[\mu_{x}^{k}\right]^{T} \mu_{x}}
\end{gathered}
$$

Likewise, the typicality can be calculated by [19]

$$
\tau^{k}(x)=1-\xi^{k}(x)=\frac{k-1}{k}-\frac{\left(\mu_{x}^{k}-x\right)^{T}\left(\mu_{x}^{k}-x\right)}{k\left[\sigma_{x}^{k}\right]^{2}}
$$

Then, the normalized eccentricity can be calculated as [19]

$$
\zeta^{k}(x)=\frac{1}{2 k}+\frac{\left(\mu_{x}^{k}-x\right)^{T}\left(\mu_{x}^{k}-x\right)}{2 k\left[\sigma_{x}^{k}\right]^{2}}<\frac{m^{2}+1}{2 k}
$$


The recursive calculation and update of the values of eccentricity and typicality result in a very efficient algorithm with very low computational effort. TEDA does not require that previously read data samples be stored in memory, only the values of $\mu$ and $\sigma$ are held, and still, there is no data loss relative to eccentricity update, since it is calculated as an exact, not approximate or learned, as the offline version presented in equation 2 . The recursive nature of TEDA enables its application to a wide range of real-time processes, where the computational resources are limited and fast response is mandatory.

TEDA is an autonomous learning procedure and calculations of eccentricity and typicality are completely data-driven. Therefore, user- or problem-specific parameters or thresholds are not required. However, fault detection approaches may be frequently seen as one-class classifiers. Thus, the task of defining the boolean membership to a certain group of data (e.g. normal or faulty) requires the definition of a threshold which, usually, does not need to be static.

A widely used principle for anomaly detection is using the so called " $n \sigma$ " thresholds [25]. However, the use of the $n \sigma$ requires the a priori strict assumption of a Gaussian distribution that TEDA tries to avoid. On the other hand, for any distribution, but, assuming a representatively large amount of independent data samples, one can use so called Chebyshev inequality [26] which states that no more than $1 / n^{2}$ of the data samples/points are more than $n \sigma$ away from the mean (where $\sigma$ denotes the standard deviation). The authors in [25] show that the condition that provides exactly the same result (but without making any assumptions on the amount of data, their independence and so on) as the Chebyshev inequality can be given as:

$$
\zeta_{k}>\frac{N^{2}+1}{2 k}
$$

\section{CASE OF STUdY}

The validation of the proposed approach based on TEDA was performed on the very well-known fault detection benchmark DAMADICS. It is one of the most used benchmarks for fault detection and diagnosis applications and is advantageous in the sense of enabling to perform the experiments using real industrial data and serving as a fair basis of comparison to other techniques.

DAMADICS is the acronym for Development and Applications of Methods for Actuator Diagnosis in Industrial Control Systems, first introduced in [27]. It is an openly available benchmark system, based on the industrial operation of the sugar factory Cukrownia Lublin SA, Poland. The benchmark considers many details of the physical and electro-mechanical properties of a real industrial actuator valve. Figures 1(a) and (b) illustrates the actuator model used in DAMADICS.

With DAMADICS, it is possible to simulate 19 abnormal events, along with the normal operation, from three actuators. A faulty state is composed by the type of the fault followed by the failure mode, which can be abrupt or incipient. Moreover, the benchmark also provides a set of online data files, each one containing the data from 24-hour use of the plant, including

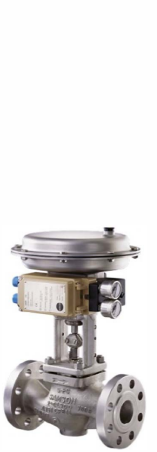

(a)

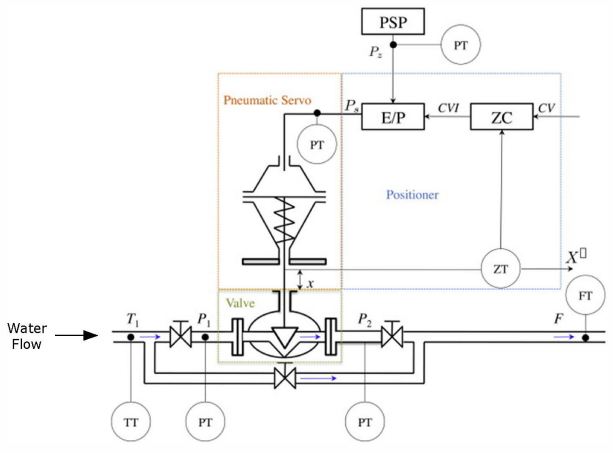

(b)
Fig. 1. Actuator model of DAMADICS benchmark [28]: (a) external view e (b) internal schema.

artificial insertion of faults in specific hours. In this paper we are working with the data from all 19 possible faults.

\section{EXPERIMENTS AND RESULTS}

The experiment consisted of applying TEDA online algorithm to the data set provided by DAMADICS benchmark. The referred data set was acquired during several days of continuous operation of the plant. It is organized in several files, each one corresponding to the plant operation of a full working day and provides data of 32 different process variables. The sampling rate is 1 data point/second, therefore, each file contains 86400 samples of each one of the 32 variables per day.

DAMADICS provides data corresponding to 25 full working days, however, only 4 of them present faulty behaviors. A total of 19 artificial faults were manually added to the process in all of its three different actuators. The faults can be classified in 4 different groups, named $f 16, f 17, f 18$ and f19, and described in Table I.

TABLE I. DESCRIPTION OF THE ARTIFICIALLY GENERATED FAULTS.

\begin{tabular}{|c|c|}
\hline Fault code & Description \\
\hline \hline f16 & Positioner supply pressure drop \\
\hline f17 & Unexpected pressure drop across the valve \\
\hline f18 & Partly opened bypass valve \\
\hline f19 & Flow rate sensor fault \\
\hline
\end{tabular}

Since the faults are located in different parts of the plant, different variables need to be selected as input to the algorithm. The feature selection or feature extraction procedure is an important stage of the task, since the set of selected features represents the overall idea of density variation. It is defined from the input/output variable space and possible preprocessing operations. It can be classified as either quantitative, such as principal component analysis (PCA) [29], partial least squares (PLS) [30], linear discriminant analysis (LDA) [31], or qualitative, for example expert systems [32] or trend modeling methods [33]. For more information on the use of feature extraction methods, the reader is referred to [34], [35], and [36].

However, in this work, we chose to use the same variables as defined in DAMADICS descriptive manual [28]. For each fault stream, the behavior of 2 input variables is analyzed. 
Likewise, the interval to be analyzed is also determined based on DAMADICS manual. Each fault stream consists of a period of time where the signals start in a normal state of operation, followed by a faulty state and, again, normal operation. The only exception is the fault stream \#13, which never leaves the faulty state once it begins.

TEDA was, then, applied to the set of 19 fault streams, and the normalized eccentricity, $\zeta$ is calculated at each time instant $k$. Therewith, each sample within the analyzed interval was classified as 'normal' or 'faulty'. The threshold $\zeta$, with $n=3$ (provides the same result as $3 \sigma$, however without making any assumption about the data distribution), thus $\zeta_{k}=5 / k$, was used for the classification.

The analysis of the obtained results considers three different measures: true positive rate (TPR), false positive rate (FPR) and total hit rate (THR) [37]. TPR is defined by

$$
T P R=\frac{n_{f}}{N_{f}} 100
$$

where $n_{f}$ is the number of correctly detected faulty samples and $N_{f}$ is the total of faulty samples.

FPR, on the other hand, is defined as

$$
F P R=\frac{n_{n}}{N_{n}} 100
$$

where $n_{n}$ is the number of normal samples incorrectly classified as faulty samples and $N_{n}$ is the total of normal samples within the designated interval.

Finally, THR is defined as

$$
T H R=\frac{n_{t}}{N_{t}} 100
$$

where $n_{t}$ is the number of correctly classified samples, both normal and faulty, and $N_{t}$ is the total of samples of the data stream.

With the application of TEDA to each of the 19 fault streams provided by DAMADICS, the values of TPR, FPR and THR were calculated. The obtained results are shown in Table II

One can observe that the application of TEDA resulted in a $73,59 \%$ total TPR, considering all 19 fault streams. Regarding the fault positive samples, the average of FPR is $15,30 \%$, resulting in $84,70 \%$ of correctly classified normal samples. In total, considering the whole data set of all 19 fault streams, the average of THR is $77,28 \%$.

Analyzing the results shown in Table II, one can observe that the higher percent rate of TEDA occurred on fault item \#7, where all faulty samples were correctly classified and, moreover, a very low FPR of $3.16 \%$ was achieved. Then, a THR of $98.90 \%$ was obtained. Similar results were obtained for many of the fault streams. Figure 2, for example, shows the obtained results for \#1.

Figure 2(a) illustrates the behavior of the 2 input variables analyzed by TEDA, where the actual beginning and end of the fault are indicated by dotted red bars. It is easy to observe the

\begin{tabular}{|c|c|c|c|c|}
\hline Item & Actuator & TPR & FPR & THR \\
\hline 1 & \multirow{7}{*}{ Actuator 1} & $92.01 \%$ & $6.70 \%$ & $92.65 \%$ \\
\hline 2 & & $83.33 \%$ & $2.90 \%$ & $93.31 \%$ \\
\hline 3 & & $36.63 \%$ & $4.50 \%$ & $75.75 \%$ \\
\hline 4 & & $0.00 \%$ & $0.00 \%$ & $47.26 \%$ \\
\hline 5 & & $72.28 \%$ & $10.43 \%$ & $88.41 \%$ \\
\hline 6 & & $73.27 \%$ & $4.75 \%$ & $90.82 \%$ \\
\hline 7 & & $100 \%$ & $3.16 \%$ & $98.80 \%$ \\
\hline 8 & \multirow{6}{*}{ Actuator 2} & $93.33 \%$ & $10.47 \%$ & $90.10 \%$ \\
\hline 9 & & $91.30 \%$ & $19.66 \%$ & $81.59 \%$ \\
\hline 10 & & $91.67 \%$ & $0.00 \%$ & $97.03 \%$ \\
\hline 11 & & $89.74 \%$ & $0.00 \%$ & $96.04 \%$ \\
\hline 12 & & $93.02 \%$ & $0.00 \%$ & $97.03 \%$ \\
\hline 13 & & $0.09 \%$ & $0.00 \%$ & $6.75 \%$ \\
\hline 14 & \multirow{6}{*}{ Actuator 3} & $80.76 \%$ & $8.28 \%$ & $88.71 \%$ \\
\hline 15 & & $68.63 \%$ & $0.00 \%$ & $92.04 \%$ \\
\hline 16 & & $83.52 \%$ & $34.55 \%$ & $73.63 \%$ \\
\hline 17 & & $83.93 \%$ & $48.28 \%$ & $60.70 \%$ \\
\hline 18 & & $93.65 \%$ & $59.20 \%$ & $54.09 \%$ \\
\hline 19 & & $71.02 \%$ & $77.78 \%$ & $43.64 \%$ \\
\hline & Mean & $73,59 \%$ & $15.30 \%$ & $77,28 \%$ \\
\hline
\end{tabular}
abrupt changes from time instant $k=58800$ to $k=59800$.
TABLE II. FAULT DETECTION RESULTS OBTAINED USING TEDA.

Such changes are immediately followed by the value of the normalized eccentricity, illustrated in Figure 2(b), exceeding, then, the value of threshold $5 / k$.

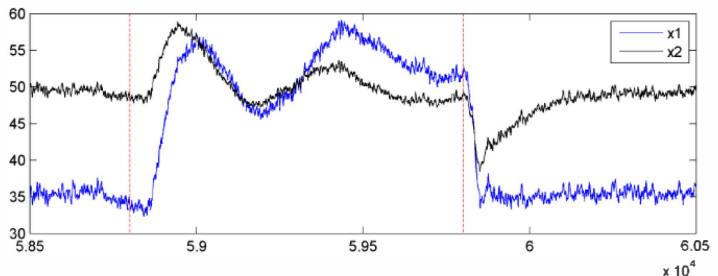

(a)

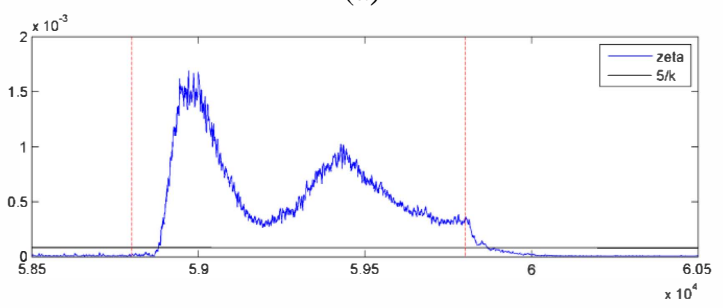

(b)

Fig. 2. results obtained for fault stream \#1: (a) input variables and (b) normalized eccentricity with threshold $5 / \mathrm{k}$.

Regarding the false positive, 6 of 19 faults resulted in FPR values of $0.00 \%$. Among them, fault stream \#12 need to be mentioned, where the obtained value of TPR is $93.02 \%$. Figure 3 shows the generated charts for such fault. By analyzing Figure 3(a) we observe that the behavior of the 2 input variables are changed at the beginning the the fault. The values of eccentricity and threshold are shown in Figure 3(b).

It is important to highlight that, the value of eccentricity increases significantly if the input data changes, particularly when such change is abrupt. Following the same construct of fault streams \#1 and \#10, Figures 4 and 5 illustrate the behavior of fault streams \#2 and \#15, respectively, including the calculated values of eccentricity and threshold. 


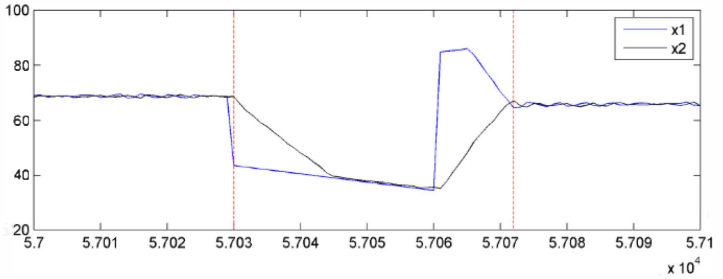

(a)

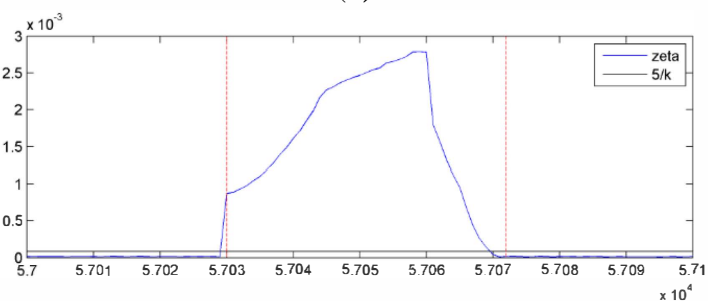

(b)

Fig. 3. results obtained for fault stream \#12: (a) input variables and (b) normalized eccentricity with threshold $5 / \mathrm{k}$.

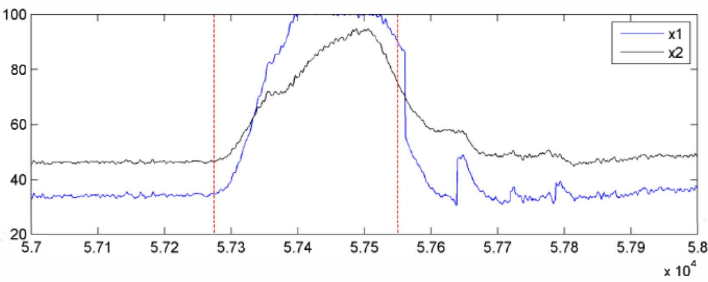

(a)

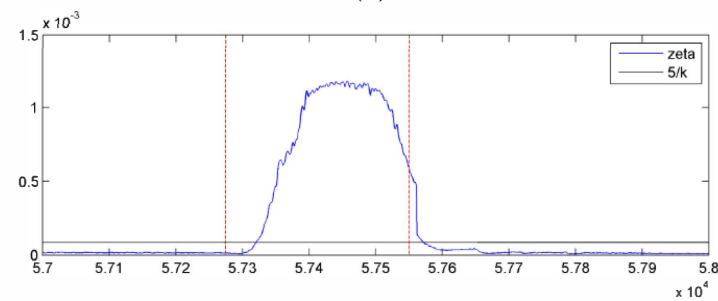

(b)

Fig. 4. results obtained for fault stream \#2: (a) input variables and (b) normalized eccentricity with threshold $5 / \mathrm{k}$.

In the case of incipient faults, i.e. faults that gradually and slowly change the values of the observed variables, the results might not be so significant. However, detecting slowly developing faults is, indeed, usually more complicated than detecting abrupt faults. Tradeoffs between early detection and false positive avoidance are more difficult to establish and, often times, slow drift faults go completely undetected because the fault detection systems assume that they are ordinary system changes and some monitoring schemes may adapt to the changes [38]. In the case of DAMADICS, fault stream \#4 was not detected as a fault since its development is very slow, as can be seen in Figure 6.

\section{CONCLUSION}

In this paper, a new approach to fault detection, based on the recently introduced typicality and eccentricity data analytics method, was proposed. The general concepts of TEDA for anomaly detection were successfully applied, for

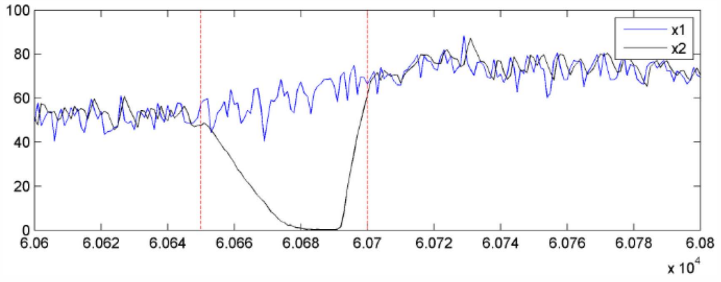

(a)

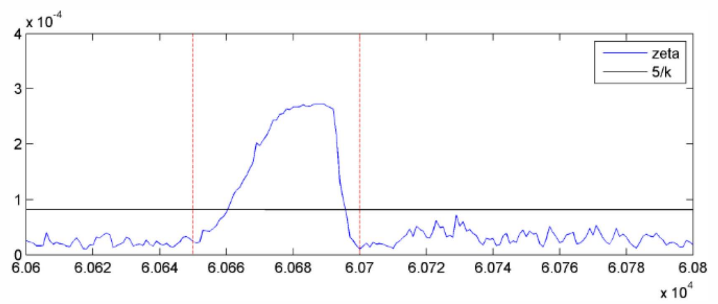

(b)

Fig. 5. results obtained for fault stream \#15: (a) input variables and (b) normalized eccentricity with threshold $5 / \mathrm{k}$.

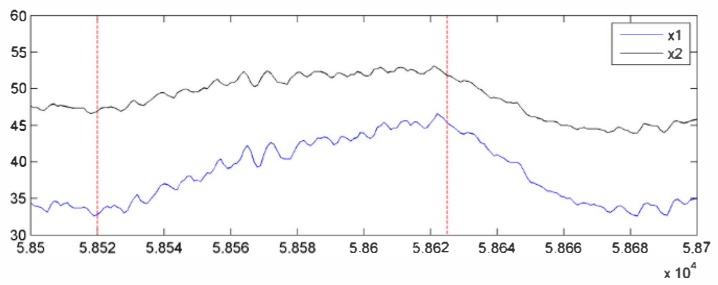

(a)

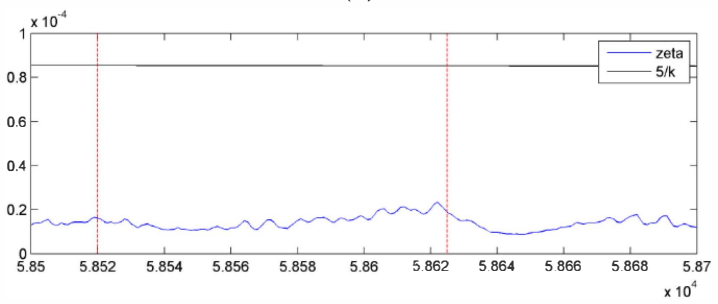

(b)

Fig. 6. results obtained for fault stream \#4: (a) input variables and (b) normalized eccentricity with threshold $5 / \mathrm{k}$.

the first time, to a very well-known real data fault detection benchmark. TEDA does not require pre-defined models, useror problem-defined parameters or thresholds or, moreover, previously known data distributions, as other standard techniques often do.

The obtained results were shown to be significant, both in hit rate and execution time. The results also showed that, the approach is suitable for industrial real-time applications, and TEDA algorithm is capable to cope with the experiment constraints and industrial environment particularities.

In our future work, special attention will be placed on the identification/classification of the types of faults by the use of evolving classification algorithms based on TEDA framework.

\section{REFERENCES}

[1] R. Isermann, Fault-Diagnosis Systems. An Introduction from Fault Detection to Fault Tolerance, 1st ed. Springer, 2006. 
[2] V. Venkatasubramanian, "Abnormal events management in complex process plants: Challenges and opportunities in intelligent supervisory control," in Foundations of Computer-Aided Process Operations, 2003, pp. 117-132.

[3] V. Venkatasubramanian, R. Rengaswamy, and S. Kavuri, "A review of process fault detection and diagnosis. part i: Quantitative model-based methods," Computers and chemical engineering, vol. 27, no. 3, pp. 293-311, 2003.

[4] B. S. J. Costa, P. Angelov, and L. A. Guedes, "Real-time fault detection using recursive density estimation," Journal of Control, Automation and Electrical Systems, vol. 25, no. 4, pp. 428-437, 2014.

[5] B. Costa, P. Angelov, and L. A. Guedes, "A new unsupervised approach to fault detection and identification," in Neural Networks (IJCNN), 2014 International Joint Conference on. IEEE, 2014, pp. 1557-1564.

[6] W. Chen and M. Saif, "Observer-based strategies for actuator fault detection, isolation and estimation for certain class of uncertain nonlinear systems," IET Control Theory \& Applications, vol. 1, no. 6, pp. 1672 1680, 2007.

[7] G. H. Li, X. J. ; Yang, "Dynamic observer-based robust control and fault detection for linear systems," IET Control Theory \& Applications, vol. 6, no. 17, pp. 2657-2666, 2012.

[8] S. Anwar and L. Chen, "An analytical redundancy-based fault detection and isolation algorithm for a road-wheel control subsystem in a steerby-wire system," IEEE Transactions on Vehicular Technology, vol. 56, no. 5, pp. 2859-2869, 2007.

[9] S. Simani and R. J. Patton, "Fault diagnosis of an industrial gas turbine prototype using a system identification approach," Control Engineering Practice, vol. 16, no. 7, pp. 769 - 786, 2008.

[10] S. Oblak, I. Skrjanc, and S. Blazic, "Fault detection for nonlinear systems with uncertain parameters based on the interval fuzzy model," Engineering Applications of Artificial Intelligence, vol. 20, no. 4, pp. $503-510,2007$

[11] S. El-Shal and A. Morris, "A fuzzy expert system for fault detection in statistical process control of industrial processes," IEEE Transactions on Systems, Man, and Cybernetics, Part C: Applications and Reviews, vol. 30, no. 2, pp. $281-289,2000$.

[12] D. F. Leite, M. B. Hell, P. C. Jr., and F. Gomide, "Real-time fault diagnosis of nonlinear systems," Nonlinear Analysis: Theory, Methods \& Applications, vol. 71, no. 12, pp. e2665 - e2673, 2009.

[13] A. T. Vemuri, M. M. Polycarpou, and S. A. Diakourtis, "Neural network based fault detection in robotic manipulators," IEEE T. Robotics and Automation, vol. 14, no. 2, pp. 342-348, 1998.

[14] C. A. Laurentys, R. M. Palhares, and W. M. Caminhas, "Design of an artificial immune system based on danger model for fault detection." Expert Syst. Appl., vol. 37, no. 7, pp. 5145-5152, 2010.

[15] C. A. Laurentys, G. Ronacher, R. M. Palhares, and W. M. Caminhas, "Design of an artificial immune system for fault detection: A negative selection approach." Expert Syst. Appl., vol. 37, no. 7, pp. 5507-5513, 2010.

[16] P. Angelov, "Anomaly detection based on eccentricity analysis," in Proc. IEEE Symposium Series in Computational Intelligence (SSCI 2014), Orlando, Florida, U.S.A., Dec. 2014.

[17] _ Autonomous Learning Systems: From Data to Knowledge in Real Time. John Willey and Sons, 2012

[18] D. Kangin and P. Angelov, "Recursive svm based on teda," in THE THIRD INTERNATIONAL SYMPOSIUM ON STATISTICAL LEARNING AND DATA SCIENCES (SLDS 2015), London, UK, April 2015, pp. 117-132, to appear, 2015.

[19] _- "New autonomously evolving classifier tedaclass," IEEE Transactions on Cybernetics, 2015, to appear, 2015.

[20] A. Hossain, Z. Choudhury, and S. Suyut, "Statistical process control of an industrial process in real time," IEEE Transactions on Industry Applications, vol. 32, no. 2, pp. 243 - 249, 1996.

[21] G. Cook, J. Maxwell, R. Barnett, and A. Strauss, "Statistical process control application to weld process," IEEE Transactions on Industry Applications, vol. 33, no. 2, pp. 454 - 463, 1997.

[22] D. Osherson and E. E. Smith, "Discussion: On typicality and vagueness," Cognition, vol. 64, pp. 189-206, 1997.
[23] P. Angelov, "Outside the box: an alternative data analytics frame-work," Journal of Automation, Mobile Robotics and Intelligent Systems, vol. 8, no. 2, pp. 29-35, 2014.

[24] _ - "Evolving takagi-sugeno fuzzy systems from data streams (ets+)." in Evolving intelligent systems : methodology and applications, ser. IEEE Press series in Computational Intelligence, P. Angelov, D. Filev, and N. Kasabov, Eds. John Wiley and Sons and IEEE Press, 2010, pp. 21-50.

[25] A. Bernieri, B. G., and L. C., "On-line fault detection and diagnosis obtained by implementing neural algorithms on a digital signal processor," IEEE Transactions on Instrumentation and Measurement, vol. 45, p. 894899, 1996

[26] J. G. Saw, M. Yang, and T. C. Mo, "Chebyshev inequality with estimated mean and variance," The American Statistician, vol. 38, no. 2, pp. 130-132, 1984.

[27] M. Bartys, R. Patton, M. Syfert, S. de las Heras, and J. Quevedo, "Introduction to the damadics actuator fdi benchmark study," Control Engineering Practice, vol. 14, no. 6, pp. 577-596, 2006.

[28] Damadics information web site. [Online]. Available: http://diag.mchtr.pw.edu.pl/damadics/

[29] A. Malhi and R. X. Gao, "Pca-based feature selection scheme for machine defect classification." IEEE T. Instrumentation and Measurement, vol. 53, no. 6, pp. 1517-1525, 2004.

[30] A. Kembhavi, D. Harwood, and L. S. Davis, "Vehicle detection using partial least squares." IEEE Trans. Pattern Anal. Mach. Intell., vol. 33, no. 6, pp. 1250-1265, 2011

[31] F. Song, D. Mei, and H. Li, "Feature selection based on linear discriminant analysis," in Proceedings of the 2010 International Conference on Intelligent System Design and Engineering Application - Volume 01, ser. ISDEA '10. Washington, DC, USA: IEEE Computer Society, 2010, pp. 746-749. [Online]. Available: http://dx.doi.org/10.1109/ISDEA.2010.311

[32] S. S. Pande and B. S. Prabhu, "An expert system for automatic extraction of machining features and tooling selection for automats," Comput.-Aided Eng. J., vol. 7, no. 4, pp. 99-103, Aug. 1990. [Online]. Available: http://dx.doi.org/10.1049/cae.1990.0024

[33] S. Dash, R. Rengaswamy, and V. Venkatasubramanian, "Fuzzy-logic based trend classification for fault diagnosis of chemical processes," Computers \& Chemical Engineering, vol. 27, no. 3, pp. 347 - 362, 2003. [Online]. Available: http://www.sciencedirect.com/science/article/pii/S0098135402002144

[34] M. J. Anzanello, Feature Extraction and Feature Selection: A Survey of Methods in Industrial Applications. John Wiley \& Sons, Inc., 2010. [Online]. Available: http://dx.doi.org/10.1002/9780470400531.eorms0321

[35] M. D. Levine, "Feature extraction: A survey," Proceedings of the IEEE, vol. 57, no. 8, pp. 1391-1407, Jun. 2005.

[36] H. Liu, G. Chen, S. Jiang, and G. Song, "A survey of feature extraction approaches in analog circuit fault diagnosis." in PACIIA (2). IEEE Computer Society, 2008, pp. 676-680.

[37] M. Grbovic, S. Vucetic, W. Li, P. Xu, and A. K. Usadi, "A boosting method for process fault detection with detection delay reduction and label denoising," in Proceedings of the First International Workshop on Data Mining for Service and Maintenance, ser. KDD4Service '11. New York, NY, USA: ACM, 2011, pp. 7-11. [Online]. Available: http://doi.acm.org/10.1145/2018673.2018675

[38] K. Goebel and W. Yan, "Correcting sensor drift and intermittency faults with data fusion and automated learning," Systems Journal, IEEE, vol. 2, no. 2, pp. 189-197, June 2008. 